\title{
Die Pankreasanastomose - ein Videovergleich unterschiedlicher Anastomosentechniken
}

\section{Pancreatic Anastomosis - A Video Comparison of Different Techniques}

\section{Zusammenfassung \\ $\nabla$}

Einleitung: Die Pankreasanastomose ist die Achillesferse der Pankreasresektion insbesondere beim weichen Pankreas. Es wurden viele unterschiedliche Techniken in der Literatur beschrieben. Zu den häufigsten angewendeten Techniken gehören die Anastomosierung des Pankreas zum Jejunum entweder als Pankreatikojejunostomie (Gang-zu-Mukosa-Anastomose) oder Pankreatojejunostomie (invaginierende Anastomose). Eine andere vielfach eingesetzte Anastomose zur Rekonstruktion ist die Nahtverbindung zum Magen: Pankreatogastrostomie. Für keine der Anastomosen konnte eine klinische Überlegenheit in Bezug auf postoperative Komplikationen (Pankreasfistel, Pankreasanastomoseninsuffizienz, postoperative Blutung und Magenentleerungsstörung) sicher nachgewiesen werden.

Indikation: Rekonstruktion nach Pankreaskopfresektion.

Operationsverfahren: Anastomose von Pankreas zum Darm bzw. Magen.

Schlussfolgerung: Es gibt keinen Goldstandard zur Durchführung der Pankreasanastomose, sodass die in der jeweiligen Klinik standardisierte Technik zum Einsatz kommen sollte.

\section{Einleitung \\ $\nabla$}

Durch Fortschritte in der Pankreaschirurgie ist die Letalität nach Pankreaskopfresektion in den letzten Jahrzehnten vor allem in spezialisierten Zentren auf unter 5\% gesunken. Dennoch ist die Morbidität, die vor allem durch Insuffizienz der pankreatoenterischen Anastomose verursacht und als Pankreasfistel klinisch manifest wird, mit 30-50\% immer noch sehr hoch. Die Pankreasanastomose ist die Achillesferse der Pankreasresektion insbesondere beim weichen Pankreas [1]. In zahlreichen Studien wurde daher versucht, die ideale Pankreasanastomose zu identifizieren, und es wurden viele unter- schiedliche Techniken in der Literatur beschrieben. Zu den häufigsten angewendeten Techniken gehören die Anastomosierung des Pankreas zum Jejunum entweder als Pankreatikojejunostomie (Gang-zuMukosa-Anastomose) oder Pankreatojejunostomie (invaginierende Anastomose) $[1,2]$. Eine andere vielfach eingesetzte Anastomose zur Rekonstruktion ist die Nahtverbindung zum Magen: Pankreatogastrostomie [3]. Für keine der Anastomosen konnte eine klinische Überlegenheit in Bezug auf postoperative Komplikationen (Pankreasfistel, Pankreasanastomoseninsuffizienz, postoperative Blutung und Magenentleerungsstörung) sicher nachgewiesen werden $[4,5]$.

\section{Anastomosentechniken der Pankreasanastomose (Video) \\ $\nabla$}

Der Patient befindet sich jeweils in Rückenlagerung, die Kamera filmt von der rechten Seite des Patienten. Die Pankreaskopfresektion ist bereits durchgeführt worden. Das Resektionsausmaß entspricht der zugrunde liegenden Pathologie (benigne, maligne, entzündlich) und ist nicht Inhalt dieses Artikels. Ausgangspunkt ist jeweils der Situs nach Abgabe des Präparats. Im Folgenden werden die einzelnen Anastomosentechniken erläutert. Dem jeweiligen Anastomosenvideo ist eine Schemazeichnung vorangestellt.

\section{Pankreatikojejunostomie}

(Gang-zu-Mukosa-Anastomose)

(M. Niedergethmann) ( $\odot$ Abb. 1 a)

Der Pankreaskorpus ist ca. 1-2 cm mobilisiert. Das proximale Jejunum ist zur Anastomosierung retrokolisch in den rechten Oberbauch verlagert worden. Die Endzu-Seit-Pankreatikojejunostomie wird in zweireihiger Nahttechnik durchgeführt. Für die innere direkte Gang-zu-MukosaNaht werden Einzelknopfnähte PDS 5-0 und für die nicht invaginierende äußere Pankreaskapsel-zu-Serosa-Naht eine fortlaufende Naht PDS 4-0 (doppelt armiert) verwendet. Die Eröffnung des Jejunums wird in der Größe passend zum Durchmesser des Pankreasgangs gewählt und mit dem elektrischen Skalpell durchgeführt. Auf eine intraduktale oder intraluminale Drainage wird verzichtet.

\section{Pankreatogastrostomie (T. Keck) (๑ Abb. 1 b)}

Die Mobilisation des Pankreas für die Pankreatogastrostomie erfolgt weiter als für die klassische Pankreato- oder Pankreatikojejunostomie. Der Pankreaskorpus wird über $4 \mathrm{~cm}$ mobilisiert. Die kranialen und kaudalen kleinen Äste zum Pankreaskorpus aus der A. lienalis und der A. mesenterica superior werden durchtrennt. Der Pankreasschwanz wird entlang der V. lienalis mobilisiert. Auf der Pankreasschnittfläche ist auf subtile Blutstillung zu achten, da Nachblutungen aus der Pankreasschnittfläche in den Magen andernfalls gehäuft auftreten können [4]. Kleinere Blutungen werden unter Beachtung des Verlaufs des Pankreasgangs mit 5-0 PDS C1 durchstochen. An der dorsalen Wand der Magens erfolgt eine $1-1,5 \mathrm{~cm}$ große Inzision. Die Magenvorderwand wird als Arbeitskanal $5 \mathrm{~cm}$ lang inzidiert. In einem Abstand von $1 \mathrm{~cm}$ um die dorsale Inzision erfolgt die Vorlage einer zirkulären Tabaksbeutelnaht mit 20 PDS SH. Das Pankreas wird an den im Vorfeld angelegten Haltenähten in den Magen invaginiert, wobei dies zur Schonung des Gewebes durch aktiven Zug der Magenhinterwand über das Pankreas und nicht durch Zug des Pankreas in den Magen erfolgen sollte. Im nächsten Schritt erfolgt die zirkuläre Einzelknopfanastomose (5 mm Abstand) zwischen Magenhinterwand und Pankreas mit 4-0 PDS SH. Nach Abschluss der Anastomose wird die Tabaksbeutelnaht zugezogen, wobei der Pankreasgang für diese Maßnahme temporär geschient wird. Abschließend wird die ventrale Gastrotomie in fortlaufender Nahttechnik mit 4-0 PDS einreihig verschlossen.

\section{Pankreatojejunostomie (invaginierende Anastomose) \\ (R. Grützmann) ( $\odot$ Abb. 1 c)}

Der Pankreaskorpus wird ca. 1-2 cm mobilisiert. Nach sorgfältiger Blutstillung der Pankreasresektionsfläche mit 4-0 Vicrylumstechungsnähten erfolgt zunächst die Aufhängung der Hinterwand des Pankreasgangs mit einzelnen 5-0 PDS-Nähten. Das erleichtert die 2. hintere Nahtreihe und hilft insbesondere $\mathrm{zu}$ vermeiden, dass der Pankreasgang durch die Anasto- 

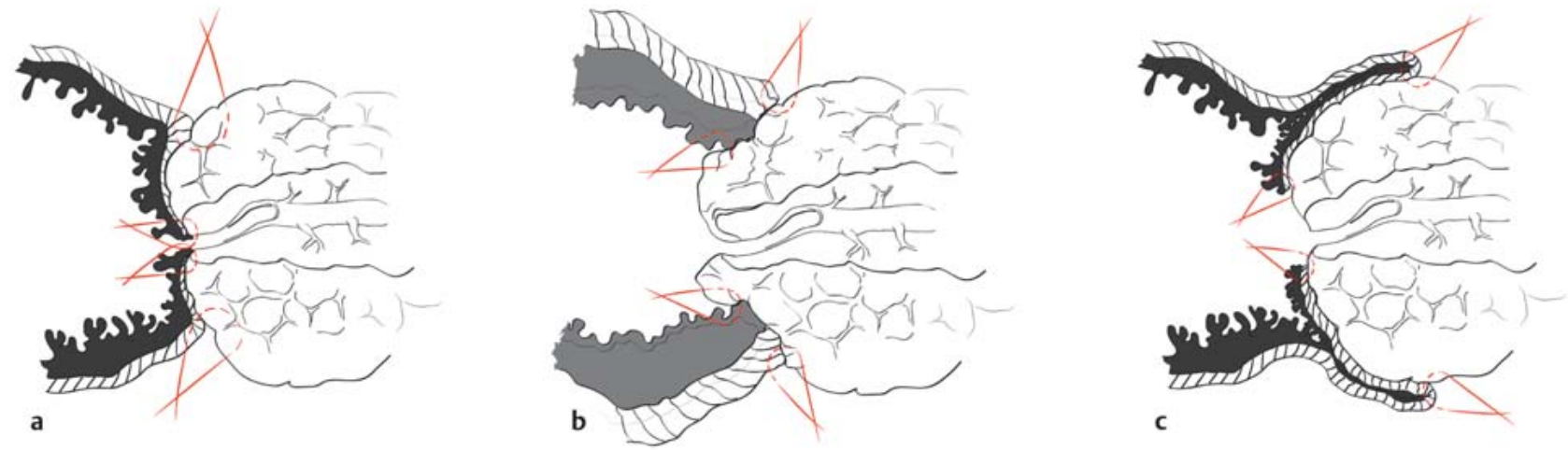

Abb. 1 a bis c Schematische Darstellung verschiedener Anastomosentechniken für die Pankreasanastomose. a Gang-zu-Mukosa-Pankreatikojejunostomie; b Pankreatojejunostomie; c invaginierende Pankreatojejunostomie.

mosennähte eingeengt oder verschlossen wird. Nachfolgend wird die äußere hintere Nahtreihe von Pankreaskapsel zum Jejunum mit 4-0 PDS vorgelegt. Darauf erfolgt das Knüpfen dieser Nähte und das Eröffnen des Jejunums ca. 2-3 mm neben der Nahtreihe üblicherweise mit dem monopolaren elektrischen Skalpell (hier bipolar, da Schrittmacherträger). Eine sorgfältige Blutstillung ist notwendig, um Nachblutungen zu verhindern und die nächsten Nähte exakt durchführen zu können. Jetzt wird die innere hintere Nahtreihe unter Einbeziehung der vorgelegten Nähte am Pankreasgang mit 4-0 vorgelegt und dann geknotet. Nun erfolgt invertierend die Naht der Vorderwand mit 4-0 PDS in Einzelknopftechnik. Wichtig ist dabei, dass die Nähte durch das Pankreas sorgsam unter Einsatz der Pinzette als Hypomochlion durchgezogen werden. Sehr leicht kann es sonst bei nicht exakter Technik zu Einrissen am Pankreas kommen, aus denen sich dann Pankreasfisteln entwickeln können. Diese Nahtreihe wird durch invaginierende Nähte der Vorderwand mittels 3-0 Vicryl gesichert und damit die Anastomose vervollständigt.

\section{Fazit}

Die Pankreasanastomose ist die Hauptursache für Komplikationen nach Pankreasresektion, insbesondere wenn der Pankreasgang eng und die Resektionsfläche weich ist. Es gibt eine Vielzahl unterschiedlicher Rekonstruktionstechniken

\section{Abstract \\ V}

Introduction: The pancreatic anastomosis seems to be the most difficult and dangerous anastomosis in general surgery, especially in a soft pancreas. Many techniques have been described. The techniques most often used are: anastomosis of the pancreas to the jejunum as a pancreatico-jejunostomy (duct-tomucosa anastomosis) or as a pancreato-jejunostomy (invaginating anastomosis). Another widely used anastomosis for reconstruction after pancreatic head resection is from the stomach to the pancreas, i.e., pancreato-gastrostomy. In literature the data concerning postoperative complications (pancreatic fistula, postoperative bleeding and others) are not consistent.

Indications: Reconstruction after pancreatic head resection.

Procedure: Anastomosis between small intestine or stomach and the pancreas.

Conclusion: There is no gold standard for pancreatic anastomosis. Thus, of the different commonly used techniques, in our opinion, the best technique for each surgeon seems to be the one that he/she is most familiar with. nach Pankreaskopfresektion. Die am häufigsten angewendeten Techniken unterscheiden sich in der Literatur nicht in Bezug auf postoperative Komplikationen (Pankreasfistel, Pankreasanastomoseninsuffizienz, postoperative Blutung und Magenentleerungsstörung). Da es bisher keinen Goldstandard zur Durchführung der Pankreasanastomose gibt, sollte die in der jeweiligen Klinik standardisierte Technik zum Einsatz kommen [6].

\section{Interessenkonflikt: Nein}

\section{Literatur}

1 Niedergethmann $M$, Dusch N, Widyaningsih $R$ et al. Risk-adapted anastomosis for partial pancreaticoduodenectomy reduces the risk of pancreatic fistula: a pilot study. World J Surg 2010; 34:1579-1586
2 Rückert F, Kersting S, Fiedler D et al. Chronic pancreatitis: early results of pancreatoduodenectomy and analysis of risk factors. Pancreas 2011; 40: 925-930

3 Wellner UF, Sick O, Olschewski M et al. Randomized controlled single-center trial comparing pancreatogastrostomy versus pancreaticojejunostomy after partial pancreatoduodenectomy. J Gastrointest Surg 2012; 16: 1686-1695

4 Bassi C, Falconi M, Molinari E et al. Duct-tomucosa versus end-to-side pancreaticojejunostomy reconstruction after pancreaticoduodenectomy: results of a prospective randomized trial. Surgery 2003; 134: 766-771

5 Yeo CJ, Cameron JL, Maher MM et al. A prospective randomized trial of pancreaticogastrostomy versus pancreaticojejunostomy after pancreaticoduodenectomy. Ann Surg 1995; 222: 580-592

6 Distler M, Grützmann R. New aspects of surgery for pancreatic cancer: Principles, results and evidence. Pathologe 2012; 33 (Suppl. 2): 258-265 
R. Grützmann ${ }^{1}$, M. Distler ${ }^{1}$, T. Keck ${ }^{2}$,

M. Niedergethmann ${ }^{3}$

${ }^{1}$ Klinik und Poliklinik für Viszeral-, Thoraxund Gefäßchirurgie, Universitätsklinikum Dresden, Deutschland

2 Klinik für Chirurgie, Universitätsklinik Schleswig-Holstein, Campus Lübeck, Deutschland

${ }^{3}$ Klinik für Allgemein- und Viszeralchirurgie, Alfried Krupp Krankenhaus Rüttenscheid, Deutschland
Der Link zum Video:

http://www.tiny.cc/zbc_Video_1_2013

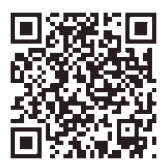

\section{Bibliografie}

DOI http://dx.doi.org/10.1055/s-1328212

Zentralbl Chir 2013; 138: 24-26

(c) Georg Thieme Verlag KG Stuttgart · New York .

ISSN 0044-409X

\section{Korrespondenzadresse}

Prof. Robert Grützmann

Klinik und Poliklinik für Viszeral-, Thorax- und Gefäßchirurgie

Universitätsklinikum Dresden

Fetscherstraße 74

01307 Dresden

Deutschland

Tel.: $0351 / 45816996$

Fax: 0351/4584395

robert.gruetzmann@uniklinikum-dresden.de 